\title{
Risk Factor Determination and Antifungal Susceptibility Patterns of Candida in Neonatal Oral Candidiasis
}

\author{
Soma Mondal ${ }^{1}$, Avijit Mondal ${ }^{2}$, Nupur Pal ${ }^{3}$ \\ ${ }^{I}$ (Department of Microbiology, College of Medicine \& JNM Hospital, WBUHS, Kalyani, India) \\ ${ }^{2}$ (Department of Physiology, College of Medicine \& JNM Hospital, WBUHS, Kalyani, India) \\ ${ }^{3}$ (Department of Microbiology, I.P.G.M.E\&R, Kolkata, India)
}

\begin{abstract}
Context: Recently Candidiasis has become one of the major nosocomial infections among hospitalized neonates. Several Candida species can give rise to same clinical presentation but their antifungal susceptibility patterns may vary.

Aim and Objectives: The aim of the present study is to assess the risk factors contributing to neonatal oral candidiasis, identification of its causative species and determination of antifungal susceptibility patterns.

Settings and Design: This case control study was performed to determine the causative species and risk factors of neonatal candidiasis among the neonates admitted to neonatal care unit.

Materials and Methods: Swabs from oral thrush of 119 neonates were collected, same number of age and sex matched neonates were examined as control. Blood culture was also performed in suspected cases of candidemia. Swabs were collected from the hands of 28 health care workers and 58 mothers and all the relevant data was collected. After doing speciation antifungal susceptibility test was performed for 94 pure isolates. Statistical analysis used: All the results were analyzed using RaoSOFT, the result was expressed as mean \pm $S D$ and $p$ value $<0.05$ was considered significant.
\end{abstract}

Keywords: Candida, neonate, oral thrush, risk factors

\section{Introduction}

Recently candidiasis has become one of the major fungal infection among hospitalized neonates. Colonization of the neonatal skin and gastrointestinal tract is the first step in the pathogenesis of invasive candidiadis ${ }^{1}$.The incidence of Candida infection is greatest in low birth weight and extremely low birth weight neonates ${ }^{2}$. Different Candida spp. have been shown to cause a similar spectrum of disease ranging from oral thrush to invasive disease, yet differences in disease severity and susceptibility to different antifungal agents have been reported ${ }^{3}$. Candida infection can occur in various ways among neonates. They acquire the infection by vertical or nosocomial route of transmission ${ }^{4,5}$. It is well known fact that Candida albicans is the most common species causing different type of candidiasis. Recent study indicate that besides Candida albicans, the four most common Candida species isolated from blood include C.parapsilosis, C. tropicalis, C. glabrata and C. krusei ${ }^{6,7}$. Various phenotypic methods are available for speciation of Candida. In the present study we have used germ tube test, Chrome agar Candida medium and Candida sugar utilisation kit for species identification of Candida. Chrome agar Candida media was found to very effective for speciation by allowing rapid and correct identification of yeast colonies and easy recognition of mixed cultures ${ }^{6,7,8}$. Recently there is a increase incidence of drug resistant Candida infection specially among patients admitted in ICCU and NICU. The study was conducted to determine risk factors of neonatal candidiasis, identifying different causative species of Candida and also to determine antifungal susceptibility pattern of the isolates. Thus it will ultimately help the physicians to plan for appropriate preventive and therapeutic measures.

\section{Materials And Methods}

The study was conducted in the department of Microbiology after receiving the approval from institutional ethical committee, at R.G. Kar Medical College Kolkata from April 2010 to March 2011. Mothers of the cases were reassured that there is no risk to their babies so far as the study is concerned. Their queries regarding the disease and the study were properly addressed. After thorough screening, 119 neonates with visible oral thrush were included in the study as cases. Swabs from visible oral thrush were collected from all the cases. Same number of age and sex matched healthy neonates without oral thrush were taken as control. Swabs were also collected from hands of all health care workers of neonatal care unit and mothers of the cases . All the samples were inoculated in Sabouraud dextrose agar (SDA) and were incubated for 24 to 48 hours at $37^{\circ} \mathrm{C}$. Any growth found on SDA slope was processed for identification of the species by several tests. Species identification of Candida was done by morphotyping on Corn meal agar ${ }^{9}$, Hi Candida Identification Kit and CHROM agar Candida media ${ }^{10}$ [HiMedia, Mumbai] were also used for species identification. CHROM agar 
Candida $^{11,12,13}$ used to differentiate several Candida species by growth of different coloured colonies in it. HiCandida Identification $\mathrm{Kit}^{13}$ was used to detect several sugar utilization properties by the Candida isolates. Antifungal sensitivity was performed on 94 pure isolates. The test was performed as per CLSI M-44A document ${ }^{14}$ using Muller Hinton Glucose Methylene Blue (MH-GMB) medium. Fluconazole $(25 \mu \mathrm{g})$ disc and amphotericin B (100 units) disc were used to test antifungal resistance of various Candida species by disc diffusion method. During the study period blood culture was also performed and for suspected cases and 14 cases of invasive candidiasis were detected among them. Species identification and antifungal susceptibility tests were also performed for invasive candidiasis. All the relevant case history and data was collected from mothers and hospital documents. After collection and completion of data, statistical analysis was done using Raosoft software. Results were expressed as mean \pm SD and $\mathrm{p}$ value $<0.05$ were considered significant. Fischer's Exact test, univariate and multivariate analysis was done to determine the risk factors of oral candidiasis.

\section{Result}

Among the total admission of 3909 neonates during the study period, oral thrush was found among 119 neonates. The frequency of oral thrush observed in the present study was 3.04\% among total admission. Vaginal mode of delivery, low birth weight, use of IV fluid, IV antibiotics, low APGAR score at birth, intubation, blood and blood product transfusion were found to be important risk factors $(\mathrm{p}<0.5)$ associated with neonatal oral thrush (Table 1). Though Candida albicans was the most common species isolated from oral thrush, many non albicans Candida species were also responsible for it.( Fig. 1) In the present study it was seen that oral thrush occurs more commonly in between 8 to 11 days of age (Table 2), whereas the incidence was much lower in neonates aged less than 3 days. Oral thrush was found to be more common in preterm $(73.1 \%)$ as compared to term neonates $(26.8 \%)$. On the basis of birth weight it was found to be common in neonates having birth weight of less than $2500 \mathrm{gm}$.(Table 3) Colonization rate in the control group was also higher in low birth weight neonates (73.6\%) as compared to neonates having birth weight of $>2.5 \mathrm{~kg}$ (Table 4). Candida could be isolated from the perianal region of $43(36.1 \%)$ cases. No yeast could be isolated from environment and inanimate objects. Candida could be isolated from the hands of $12.06 \%(7 / 58)$ mothers of infected neonates and $10.7 \%$ $(3 / 28)$ health care workers of neonatal care units. In the oral thrush samples highest rate of fluconazole resistant was found in Candida glabrata (17.6\%), followed by Candida tropicalis (14.8\%), and Candida albicans $(6.06 \%)$. Out of the 14 cases of invasive candidiasis, 6 were caused by Candida tropicalis (42.8\%), 4 by Candida glabrata (28.5\%), 2 each by Candida albicans and Candida parapsilosis (14.2\%). Among blood culture positive cases of Candida, fluconazole resistant rate was found to be highest in Candida glabrata $(50 \%)$ followed by Candida tropicalis $(33.3 \%)$. All the isolates were sensitive to amphotericin B.

\section{Figures and Tables}

Table 1: Factors associated with oral thrush in Case and Control group

\begin{tabular}{|c|c|c|c|}
\hline Parameter & $\begin{array}{c}\text { Study } \\
n=(119)\end{array}$ & $\begin{array}{l}\text { Control } \\
n=(119)\end{array}$ & p value \\
\hline \multicolumn{3}{|l|}{ Sex } & \multirow{3}{*}{0.795} \\
\hline Male & $66(55.4 \%)$ & $63(52.9 \%)$ & \\
\hline Female & $53(44.5 \%)$ & $56(47.1 \%)$ & \\
\hline \multicolumn{3}{|l|}{ Para } & \multirow{3}{*}{0.513} \\
\hline Primigravida & $54(45 . \%)$ & $48(40.3 \%)$ & \\
\hline Multigravida & $65(54.6 \%)$ & $71(59.7 \%)$ & \\
\hline \multicolumn{3}{|l|}{ Delivery } & \multirow{3}{*}{$0.019^{*}$} \\
\hline Normal delivery & $69(57.9 \%)$ & $50(42.01 \%)$ & \\
\hline Caesarean section & $50(42.01 \%)$ & $69(57.9 \%)$ & \\
\hline \multicolumn{3}{|l|}{ Gestational Age } & \multirow{3}{*}{0.08} \\
\hline Term & $30(25.2 \%)$ & $90(75.6 \%)$ & \\
\hline Preterm & $89(74.7 \%)$ & $29(24.3 \%)$ & \\
\hline APGAR <5 At 1 Min & $42(35.2 \%)$ & $25(21 \%)$ & $0.002^{*}$ \\
\hline \multicolumn{4}{|l|}{ Treatment Given } \\
\hline IV fluid & $109(91.5 \%)$ & $90(75.6 \%)$ & $0.001^{*}$ \\
\hline Intubation & $18(15.1 \%)$ & $1(0.84 \%)$ & $0.0001^{*}$ \\
\hline IV Antibiotics & $108(90.7 \%)$ & $85(71.4 \%)$ & $0.0001^{*}$ \\
\hline Phototherapy & $29(24.3 \%)$ & $24(20.1 \%)$ & 0.53 \\
\hline $\begin{array}{l}\text { Blood and blood } \\
\text { product transfusion }\end{array}$ & $11(9.2 \%)$ & 0 & $0.001^{*}$ \\
\hline
\end{tabular}

Table 2: Age in days when thrush is detected

\begin{tabular}{|cccccc|}
\hline Day & $\mathbf{0 - 3}$ & $\mathbf{4 - 7}$ & $\mathbf{8 - 1 1}$ & $\mathbf{1 2 - 1 4}$ & $>\mathbf{1 4}$ \\
\hline No. of Cases & $7(5.8 \%)$ & $28(23.5 \%)$ & $36(30.25 \%)$ & $28(23.5 \%)$ & $20(16.8 \%)$ \\
\hline
\end{tabular}


Table 3: Distribution of oral thrush in relation to birth weight

\begin{tabular}{|c|c|c|c|c|}
\hline & & \multicolumn{3}{|c|}{ Birth Weight } \\
\cline { 3 - 5 } Group & Studied & $<\mathbf{1 . 5} \mathbf{~ K g}$ & $\mathbf{1 . 5}-\mathbf{2 . 4} \mathbf{~ K g}$ & $\square \mathbf{2 . 5} \mathbf{~ K g}$ \\
\hline Case & 119 & $27(22.68 \%)$ & $60(50.42 \%)$ & $32(26.89 \%)$ \\
\hline
\end{tabular}

Table 4: Distribution of colonized controls in relation to birth weight

\begin{tabular}{|c|c|c|c|c|c|}
\hline \multirow[b]{2}{*}{ Group } & \multirow[b]{2}{*}{ Studied } & \multirow[b]{2}{*}{ Colonized } & \multicolumn{3}{|c|}{ Birth Weight } \\
\hline & & & $<1.5 \mathrm{Kg}$ & $1.5-2.4 \mathrm{Kg}$ & $\square 2.5 \mathrm{Kg}$ \\
\hline Control & 119 & $38(31.93 \%)$ & $7(18.42 \%)$ & $\begin{array}{c}21 \\
(55.26 \%)\end{array}$ & $\begin{array}{c}10 \\
(26.31 \%)\end{array}$ \\
\hline
\end{tabular}

Figure 1

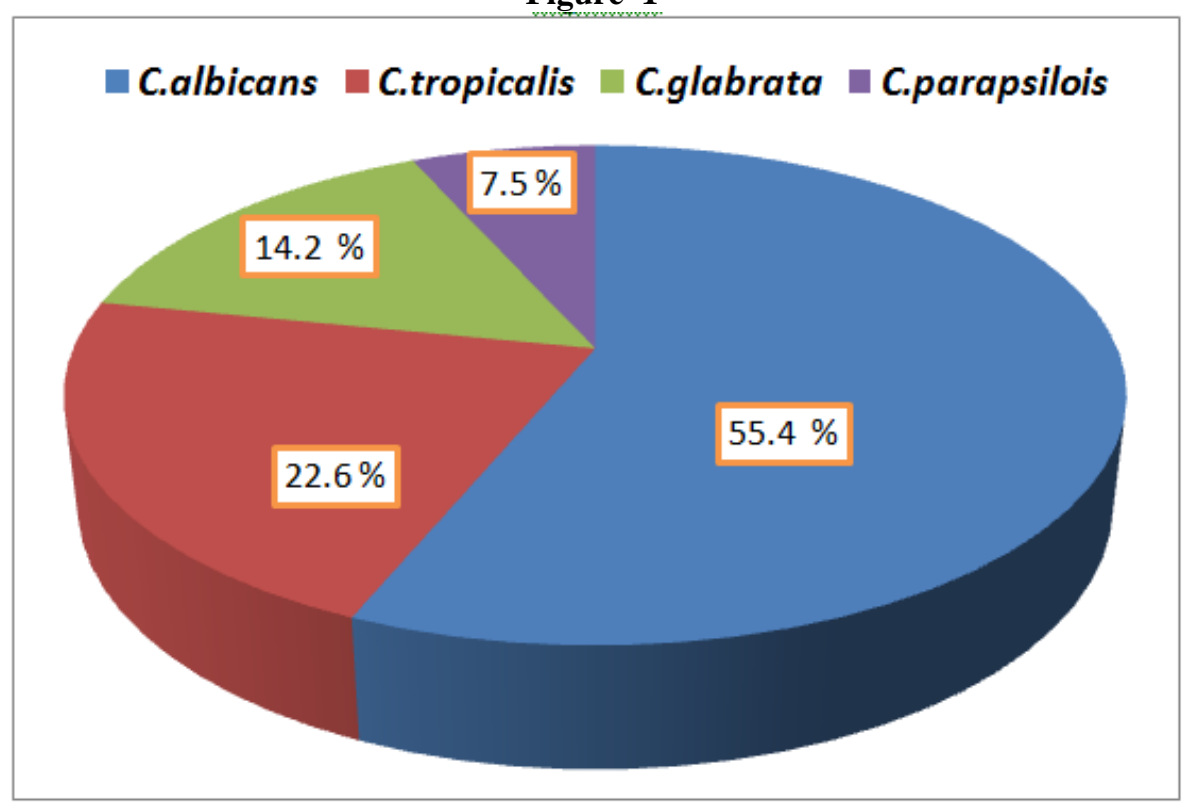

IV. Discussion

Acute pseudomembranous candidiasis is the most common type of fungal infection in neonates. The frequency of oral thrush $(3.04 \%)$ observed in the present study was close to that reported by Gupta P. et al $(3.2 \%)^{15}$. The variation of prevalence rate depends on various factors like hygiene practice in neonatal care unit, early discharge of babies from hospital, lack of regular inspection of the mouth of neonates etc. In the present study it was found that oral thrush is more common in the neonates aged 8-11days. In the present study a male preponderance was observed which was also reported by Gupta P. et al ${ }^{15}$, but others are of different opinion ${ }^{16}$. The study also shows that oral thrush is significantly more common in neonates born by normal delivery $(59.7 \%)$, similar finding was also reported by other authors ${ }^{16}$. However, another study shows that neonates born by Caesarean sections are equally prone to develop oral candidosis ${ }^{16}$. Again low birth weight, use of IV fluid, IV antibiotics, APGAR score $<5$ at 1 minute, intubation, vaginal mode of delivery, blood and blood product transfusion were found to be significantly associated with neonatal oral thrush. By performing multiple logistic regression analysis, it was found that gestational age and birth weight were the only significant risk factors responsible for oral thrush.

The results of the present study are in agreement with the study by others ${ }^{17}$. Candida colonization rate in oral cavity among control group was found to be $31.9 \%$, and it was higher in neonates with low birth weight $(73.6 \%)$. Candida could be isolated from the perianal region of $43(36.1 \%)$ cases. From the data it is clear that Candida can colonize in multiple anatomical sites of the body at a time that is with agreement with another study ${ }^{17}$. In the present study no yeast could be isolated from environment or inanimate objects. Candida could be isolated from the hands of $10.7 \%$ health care workers and $12.06 \%$ of the mothers of infected cases. Study using DNA fingerprinting techniques has shown that the both vertical and horizontal transfer contributes to colonization of Candida in very low birth weight babies in the NICU. ${ }^{18}$ Some authors have reported that high yeast carriage by health care workers of a tertiary care hospital, that may be a source of nosocomial candidiasis $^{19}$. Hand carriage rate among health care workers in seven surgical ICU across in USA have been found to be of around $30 \%{ }^{20}$. The species of Candida causing oral thrush and colonization in this study were similar to that reported by others ${ }^{17}$.Two earlier Indian studies, however have documented higher isolation rates of C. tropicalis, C. pseudotropicalis and C. stellatoidea from oral specimens ${ }^{16,21}$. In the present study a higher 
rate of fluconazole resistance was seen in non albicans Candida species (32.4\%) compared to Candida albicans $(6.06 \%)$. All the isolates were sensitive to amphotericin B, this is in agreement with the study by Mendiratta D.K et $\mathrm{al}^{17}$. They reported that the resistance to fluconazole by $C$. albicans isolated from oral cavity of neonates was $18.9 \%$ and both to fluconazole and ketoconazole was $10.4 \%$. Deorukhkar S.C et al. ${ }^{13}$ in their study have shown that resistant rate of Candida to fluconazole and amphotericin B was $27.3 \%$ and $5.8 \%$ respectively. This study depicts Candida tropicalis was the commonest species (44.4\%), isolated from blood samples, as seen in different studies ${ }^{21,22}$. It is clear from the study that Candida albicans is still the commonest pathogen causing oropharyngeal candidiasis, but in case of invasive candidiasis non albicans Candida species are the mainly responsible, those are highly resistant to fluconazole. The antifungal sensitivity pattern of Candida that was isolated from blood and colonized site (mouth, rectum) was identical in $64.2 \%(9 / 14)$ neonates, indicating the probable site of entry. Prior colonization of mucous membrane has been reported to be an important risk factor for invasive candidiasis by several authors ${ }^{17,23}$. In a recent study we found that $80 \%$ Candida spp. colonization among neonates and the most frequent oral manifestations were white, soft plaques resembling pseudomembranous candidiasis that can potentially lead to invasive candidiasis ${ }^{24}$. Among the different methods disc diffusion method is one of the simple, cost effective method that can give sensitivity result within $42 \mathrm{hrs}$. In two other studies, it was seen that prepared MH-GMB agar plates provide acceptable performance for disk diffusion testing for at least 30 days when stored at $5^{\circ} \mathrm{C}^{25,26}$.

Distinction between species facilitates the understanding regarding the reservoir and mode of transmission Candida infection. The knowledge will help to take effective measures to prevent and control the transmission of resistant pathogens. The present study will help not only to find out the prevalence of oral candidiasis in neonates, but also to identify the risk factors and the probable source of infection of neonatal candidiasis. Every possible measure should be taken to prevent nosocomial infection among the neonates. Hands of all the health care providers should be regularly examined to rule out any fungal infection.

\section{Conclusion}

Oral thrush and colonization of Candida is very common in preterm low birth weight neonates. As we know from other studies that colonization is the first step of invasive candidiasis, so it needs attention especially in neonates with identified risks. By choosing appropriate antifungal drug according to the sensitivity result for treatment will be helpful to reduce the morbidity and mortality in neonates suffering from candidiasis. Further study including large number of cases from different age groups and antifungal susceptibility tests against newer antifungal drugs can be carried out to get more information regarding this.

\section{Acknowledgement}

We acknowledge the dynamic wisdom and caring guidance of Prof. Parthajit Banerjee and Prof. Chitrita Chatterjee. Their constant encouragement, whole hearted cooperation and valuable technical guidance helped us to complete this arduous task. We are very much grateful to the West Bengal University of Health Sciences for providing us a good academic and research environment.

\section{References}

[1]. Pittet D, Monod M, Suter PM, Frenk E, Auckenthaler R. Candida colonization and subsequent infection in critically ill surgical patients. Ann Surg 1994; 220:751-8.

[2]. Singh K, Chakrabarti A, Narang A, Gopalan S. Yeast colonization and fungemia in preterm neonate in tertiary care centre. Indian J Med Res 1999; 110:169-73.

[3]. Sandra S. Richter, Rudolph P. Galask, Shawn A. Messer, Richard J. Hollis, Daniel J. Diekema, Michael A. Pfaller. Antifungal susceptibilities of Candida and epidemiology of recurrent cases. J. clin. microbiol 2005; 43:2155-61

[4]. Reef S.E, B.A. Lasker, D.S.Butcher, M.M. McNeil, H.Keyserling, and W.R.Jarvis. Nonperinatal nosocomial transmission of Candida albicans in a neonatal intensive care unit: prospective stuby. J.Clin Microbiol 1998; 36:1255-59

[5]. Waggnoner-Fountain L.A., Walker M.W., Hollis R.J., Pfaller M.A., Ferguson JE( 2), Wenzel R.P., Donowitz L.G. et al. Vertical and horizontal transmission of unique Candida species to premature newborn. Clin.Infect.Dis1996;22: 803-8

[6]. Mohammadi F, Javaheri MR, Nekoeian S, Dehghan P. Identification of Candida species in the oral cavity of diabetic patients.* Curr Med Mycol, 2016 Jun, 2(2): 1-7

[7]. Batool Sadeghi Nejad ${ }^{1,2 *}$, Ablollah Rafiei ${ }^{1}$ and Fereshteh Moosanejad. Prevalence of Candida species in the oral cavity of patients with periodentitis . African Journal of Biotechnology April, 2011.10(15): 2987-90.

[8]. Manikandan, C, Amsath, A. Isolation and rapid identification of Candida species from the oral cavity. J. Pure Appl. Zool.,20131(2): 172-77,

[9]. Chander J. Candidiasis. In: A text book of Medical Mycology, $3^{\text {rd }}$ ed. New Delhi: Mehta Publishers; 2009. P.266-90.

[10]. Baradkar VP, Mathur M, Kumar S.Hi CHROM Candida agar for identification of Candida species.Ind J. Patho Micro 2010;53:93-5.

[11]. Murray CK, Beckius ML, Green JA, Hospenthal DR. Use of chromogenic medium for the isolation of yeasts from clinical specimens. J Med Microbiol 2005; 54: 981-5.

[12]. Peng CF, Lee K-M, Lee S-H. Characterization of two chromogenic media of Candida ID and CHROM agar Candida for Preliminary identification of yeasts. J Biomed Lab Sci. 2007; 19 (2):63-8

[13]. Deorukhkar SC, Saini S. Non albicans Candida species: its isolation pattern, species distribution, virulence factors and antifungal susceptibility profile. Int J Med Sci Public Health 2013;2(3):511-6 
[14]. Clinical and Laboratory Standards Institute. Method for antifungal disk diffusion susceptibility testing of yeasts: approved standard. Wayne, PA: Clinical and Laboratory Standards Institute; CLSI M44-A; 2006

[15]. Gupta P, Faridi MM, Rawat S, Sharma P. Clinical profile and risk factors for oral candidosis in sick newborns. Indian Pediatr1996;33:299-3.

[16]. Sharma NL, Gupta SP, Philip E, Goel KM. Neonatal candidosis. Indian J Pediatr 1965;32:157-2.

[17]. Mendirratta DK, Rawat V, Thamke D, Chaturvedi P. Chhabra S, Narang P. Candida colonization in preterm babies admitted to neonatal intensive care unit in the rural setting. Indian J Med Microbiol 2006;24(4):263-7.

[18]. Bliss JM, Basavegowda KP, Watson WJ,Sheikh AU, Ryan RM. Vertical and horizontal transmission of Candida albicans in very low birth weight infants using DNA fingerprinting techniques. Pediatrics Infects Dis J 2008;27: 231-5.

[19]. Yildirim M, Sahin I, Kucukbayrak A, Ozdemir D, Tevfik Yavuz M, Oksuz S, Cakir S. Hand carriage of Candida species and risk factors in hospital personnel. Mycoses 2007; 50:189-92.

[20]. Rangel-Frausto M. S, T. Wiblin, H. M. Blumberg, Saiman L, Patterson J, Rinaldi M et al. National Epidemiology of Mycoses Survey (NEMIS): Variations in rates of bloodstream infections due to Candida species in seven surgical intensive care units and six neonatal intensive care units. Clin. Infect Dis 1999; 29:253-8.

[21]. Kaul KK, Shah JP, Pohowalla JN. Oral moniliasis in the newborn and neonatal morbidity. Indian J Pediatr 1960;27:115- 4

[22]. Shivaprakasha S, Radhakrishnan K, Karim P. Candida spp. other than Candida albicans. A major cause of fungaemia in a tertiary care centre. Indian J Med Microbiol 2007;25:405-7.

[23]. Xess I, Jain N, Hasan F, Mandal P, Banerjee U. Epidemiology of candidemia in a tertiary care centre of North India: 5-year study. Infection 2007; 35:256-9.

[24]. José Endrigo Tinoco-Araujo; Diana Ferreira Gadelha Araújo; Patrícia Gomes Barbosa ${ }^{\mathrm{I}}$; Paulo Sérgio da Silva Santos; Ana Myriam Costa de Medeiros ${ }^{\text {II }}$ Invasive candidiasis and oral manifestations in premature newborns. Einstein (São Paulo) 2013; 11 (1): 71-5

[25]. Lee SC, Fung CP,Lee N, See LC, Huang JS, Tsai CJ, Chen KS, Shieh WB. Fluconazole Disk Diffusion Test with Methylene Blueand Glucose-Enriched Mueller-Hinton Agar for Determining Susceptibility of Candida Species .J of Clin Microbiol 2001;39:1615-7

[26]. Pfaller M. A, Boyken L, Messer S.A, Hollis RJ, Diekema DJ. Stability of Mueller-Hinton Agar Supplemented with Glucose and Methylene Blue for Disk Diffusion Testing of Fluconazole and Voriconazole. J of Clin Microbiol 2004; 42:1288-89. 